\title{
Re-Evaluation of the Online Presence of Burn Centers in Austria, Germany and Switzerland
}

\author{
Benedikt S, Tuca AC, Palaĉkić A, Wurzer P, Kamolz LP
}

Division of Plastic, Aesthetic and Reconstructive Surgery, Department of Surgery, Medical University of Graz

\section{Introduction}

Websites serve as information and communication platforms and represent important tools for the self-promotion of hospitals. In 2010 Selig et al. ${ }^{1}$ evaluated the online presence of burn centers in Germany, Austria and Switzerland based on 36 quality criteria. They found that most websites provided a good overview of their services, however, burn related-information, layout and usability needed improvement.

The aim of this study was to re-evaluate the websites to assess their development over the past 6.5 years.

\section{Methods}

Within a period of 6 weeks, the websites of the German-speaking burn centers were re-evaluated according to the 36 quality criteria of Selig et al. ${ }^{1}$ divided into the following categories: General information, Information transfer, Research, Teaching, Patient care and Important aspects of clinical treatment. As several criteria were not clearly defined, we specified them to ensure clear, meaningful and standardized results.

In addition, the implementation of social media platforms was investigated.

\section{Results}

Forty-four burn units of 41 hospitals presented on 41 different websites were further investigated. Overall, an increase of quality of the websites compared to the first evaluation was noted, especially in the categories of "teaching" and "patient care". Burn-specific information was found to be still sparse.

The criteria "Introduction of staff members", "Introduction of the ward", "General information of the hospital", "Integration of multimedia based elements for patients in general", "Hyperlinks", "Means of communications", "Search aid", "Information for patients and relatives" and "Emergency numbers" were found in all cases.

Social media platforms were involved in 23/44 cases.
Distribution of the scores reached by the

single burn units (the maximum score was 41 as in five criteria 0-2 points could be allocated)

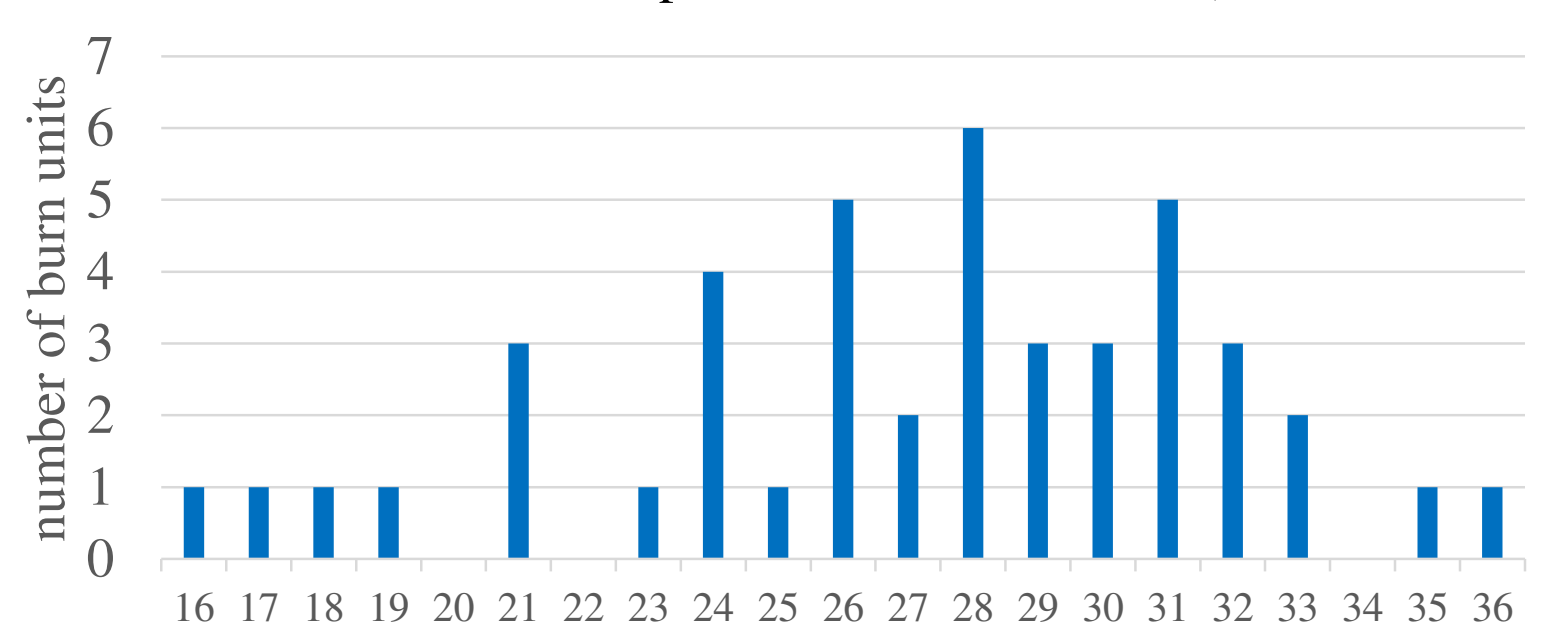

Percentage of burn units that met the criteria

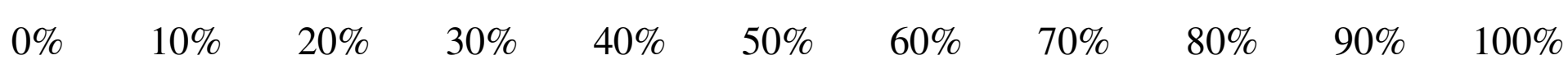

Key scientific aspects in Burn Surgery Publication or scientific projects in Burn Surgery Information on current courses Information on clinical clerkships or rotations for students Information on the preparation for medical examination Emergency number(s)

Total available number of ICU beds for burn victims Information on first aid measurements for burn injuries Background information on burn wound depth Background information on burn severity Information on treatment options Information on technical and diagnostic equipment

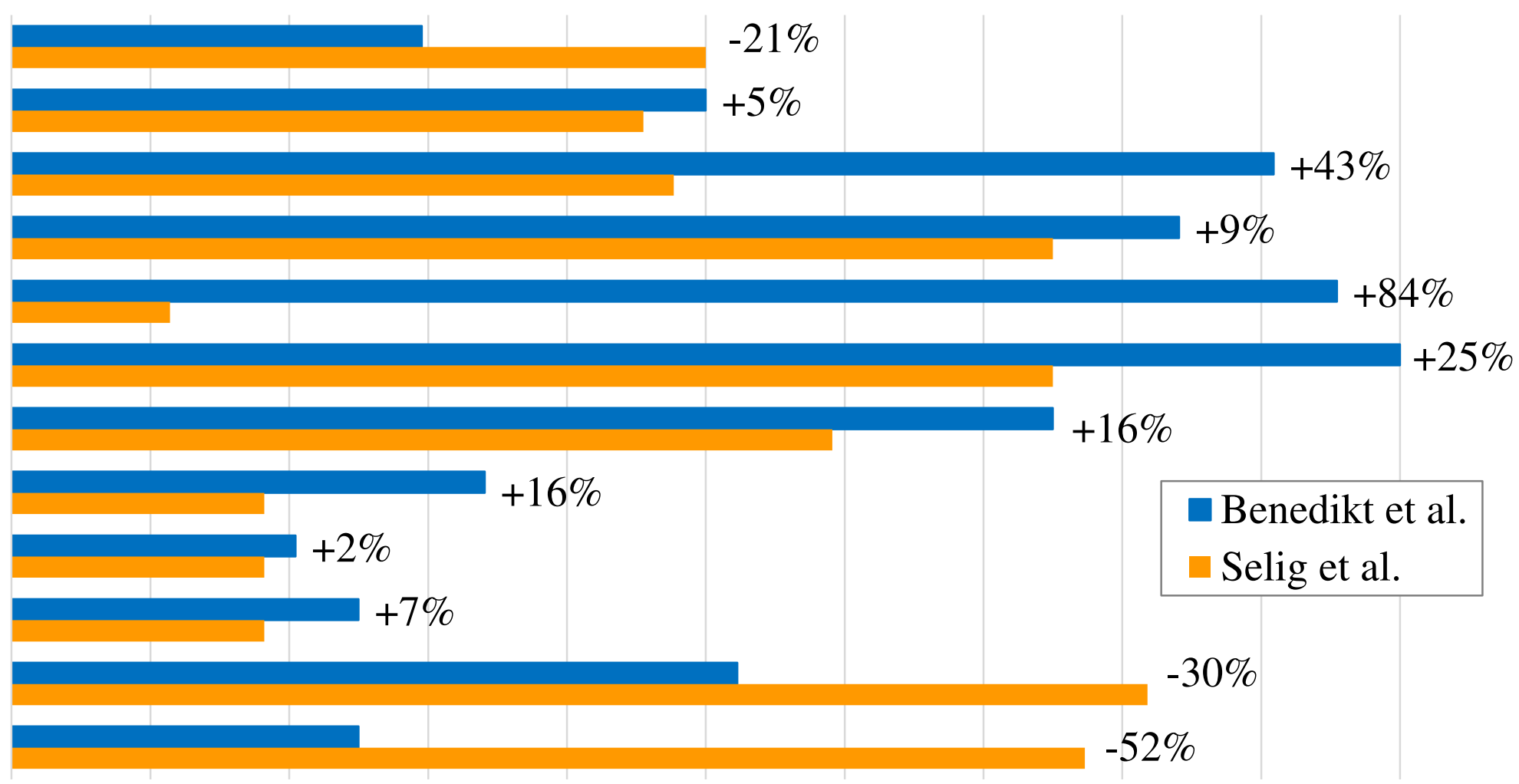

\section{Conclusion}

- Overall, the online-presence of German-speaking burn centers in Austria, Germany and Switzerland have improved over the last 6.5 years.

- Especially burn related information was still found to be insufficient.

- As the internet rapidly grows in importance, the websites should be developed further.

- The frequent implementation of links to social media platforms suggests that these have become an important medium in self-promotion.

1. Selig HF, Lumenta DB, König C, Andel H, Kamolz LP. Evaluation of the online-presence (homepage) of burn units/burn centers in Germany, Austria and Switzerland. Burns 2012;38:444-9. 